\title{
Comparação da eficácia da ropivacaína $1 \%$ quando associada ou não à hialuronidase na anestesia peribulbar para cirurgia de catarata
}

\author{
A comparison of $1 \%$ ropivacaine efficacy when associated or not with hyaluronidase in \\ peribulbar anaesthesia in cataract surgery
}

\author{
Hélio Francisco Shiroma ${ }^{1}$ \\ Efrígenes de Melo Ferreira ${ }^{2}$ \\ David Leonardo Cruvinel Isaac ${ }^{2}$ \\ Vinícius Coral Ghanem ${ }^{2}$ \\ Carlos Eduardo Leite Arieta ${ }^{3}$
}

\begin{tabular}{|c|}
\hline RESUMO \\
\hline $\begin{array}{l}\text { Objetivo: Avaliaro tempo de instalação e a qualidade da anestesia peribulbar } \\
\text { com ropivacaína } 1 \% \text {, quando associada ou não à hialuronidase para realiza- } \\
\text { ção de cirurgia de catarata. Métodos: Por meio de um ensaio clínico con- } \\
\text { trolado, foram estudados } 57 \text { pacientes, submetidos à extração extracapsular } \\
\text { da catarata sob anestesia peribulbar, distribuídos aleatoriamente em dois } \\
\text { grupos. Grupo C: ropivacaína } 1 \% \text { com hialuronidase } 100 \mathrm{UTR} / \mathrm{ml} \text {, e GrupoS: } \\
\text { apenas com ropivacaína } 1 \% \text {. Foram avaliados o tempo de instalação do } \\
\text { bloqueio de } 2 \text { em } 2 \text { minutos, utilizando-se a acinesia pela escala da motilidade } \\
\text { ocular de Nicoll, intensidade da dor durante o procedimento e a necessidade } \\
\text { de complementação anestésica. O bloqueio foi considerado satisfatório } \\
\text { quando apresentava valor } \leq 3 \text { na escala de Nicoll. Resultados: O tempo } \\
\text { médio de instalação do bloqueio no grupo C foi de } 4,07 \text { minutos ( } \pm 3,24) \text { ao } \\
\text { passo que no grupo S foi de } 5,03 \text { ( } \pm 3,28) \text { não havendo diferença estatisti- } \\
\text { camente significativa entre os grupos. Os dois grupos foram similares em } \\
\text { relação à escala de dor, com ausência de dor em } 57,14 \% \text { no grupo Ce } 68,97 \% \\
\text { no grupo S. Houve necessidade de complementação em } 5 \text { casos }(2 \text { pacientes } \\
\text { no grupo C e } 3 \text { pacientes no grupo } S \text { ). Foram constatados dois casos de } \\
\text { bradicardia intra-operatória, havendo necessidade do uso de atropina em um } \\
\text { deles. Conclusão: A ropivacaína } 1 \% \text { proporcionou boa qualidade anestésica } \\
\text { para cirurgia de catarata, apresentando discreta redução no tempo de insta- } \\
\text { lação do bloqueio motor quando associado a hialuronidase } 100 \text { UTR/ml, } \\
\text { porém sem diferença significativa. }\end{array}$ \\
\hline
\end{tabular}

Descritores: Extração de catarata; Ropivacaína/administração \& dosagem; Hialuronoglucosaminidase/administração \& dosagem; Anestesia local/métodos; Estudo comparativo; Eficácia

${ }^{1}$ Médico Anestesiologista e Ex-residente de Oftalmologia da Universidade Estadual de Campinas - UNICAMP. 2 Ex-residente de Oftalmologia da Universidade Estadual de Campinas - UNICAMP.

${ }^{3}$ Professor Assistente Doutor da Disciplina de Oftalmologia da Universidade Estadual de Campinas UNICAMP.

Endereço para correspondência: Rua Capitão Romualdo de Barros, 694, apto. 501 Bloco C - Florianópolis (SC) CEP 88040-600

E-mail: helioshiroma@hotmail.com

Recebido para publicação em 28.05.2001

Aceito para publicação em 23.04.2002

\begin{abstract}
INTRODUÇÃ̃O
A ropivacaína é um anestésico local do tipo aminoamida, com estrutura molecular semelhante ao da bupivacaína. Desenvolvida na forma de um enantiômero puro, com ação periférica e central prolongada, apresentando menor toxicidade cardíaca e no sistema nervoso central, quando comparada a bupivacaína ${ }^{(1-3)}$.

As manifestações neurológicas surgem em concentrações menores do que as necessárias para as repercussões cardíacas. Entre elas observam-se transtornos visuais e auditivos, hipoestesia perioral, vertigem, parestesias e espasmos musculares, podendo culminar com convulsões tônico-clônicas generalizadas $^{(2)}$.
\end{abstract}


Entre as alterações cárdio-vasculares encontram-se: diminuição da condução do estímulo, inotropismo negativo, arritmias e, eventualmente, parada cardíaca ${ }^{(2)}$.

A hialuronidase é uma enzima, geralmente extraída do testículo de bovinos, capaz de modificar a permeabilidade do tecido conjuntivo mediante a hidrólise do ácido hialurônico. Age diminuindo a viscosidade do cimento intercelular, promovendo uma maior difusão do anestésico injetado, o que facilita sua absorção, acelerando o início da anestesia. A adição da hialuronidase ao anestésico local para esta finalidade não é unânime ${ }^{(4-5)}$, por ser uma substância antigênica, pode levar a produção de anticorpos quando inoculado de forma repetitiva e uma superdosagem a um edema local, urticária, hipotensão e vômitos.

Este estudo teve como objetivos: 1)verificar se há variação no tempo de instalação, e 2) melhora na qualidade do bloqueio peribulbar com ropivacaína $1 \%$ associada ou não à hialuronidase $100 \mathrm{UTR} / \mathrm{ml}$ para realização da cirurgia de catarata.

\section{MÉTODOS}

Após aprovação do Comitê de Ética da Faculdade de Ciências Médicas da Universidade Estadual de Campinas - Unicamp, e consentimento informado dos pacientes, foi realizado um estudo prospectivo, duplo cego, em 57 pacientes estado físico I a III (segundo a classificação da ASA), submetidos à extração extracapsular da catarata em regime ambulatorial.

Os pacientes foram distribuídos aleatoriamente em dois grupos: grupo C (ropivacaína associada à hialuronidase $100 \mathrm{UTR} / \mathrm{ml}$ ) e o grupo S (ropivacaína sem hialuronidase) (Quadro 1), e submetidos à anestesia peribulbar, utilizando a técnica de injeção dupla com agulha $25 \times 7 \mathrm{~mm}$, sendo administrado $4 \mathrm{ml}$ (temporal inferior com inclinação súpero-medial de cerca de 15 graus) e $3 \mathrm{ml}$ (nasal-superior), de uma solução anestésica, preparada sem o conhecimento do oftalmologista que realizou o bloqueio.

Após a realização do bloqueio, utilizou-se um peso de mercúrio de $600 \mathrm{~g}$, e teve início a avaliação da motilidade ocular de cada músculo reto separadamente, em intervalos regulares de dois minutos até o décimo minuto, seguindo a escala de Nicoll (Quadro 2). Ao final de cada intervalo eram somadas as notas dos músculos com um placar que poderia variar entre $0 \mathrm{a}$

\begin{tabular}{|ll|}
\hline & Quadro 1. Grupos dos pacientes \\
Grupo C & Ropivacaína 1\% com Hialuronidase 100 UTR/ml \\
Grupo S & Ropivacaína 1\% \\
\hline
\end{tabular}

Quadro 2. Escala de Nicoll: motilidade de cada músculo extra-ocular

\begin{tabular}{ll} 
Normal & 2 \\
Diminuição da motilidade & 1 \\
Acinesia & 0 \\
\hline
\end{tabular}

\begin{tabular}{|cc|}
\hline & Quadro 3. Escala analógica de dor de $\mathbf{0}$ a $\mathbf{1 0}$ \\
Escore & Intensidade da dor \\
0 & Sem dor \\
5 & Dor moderada \\
10 & Dor insuportável \\
\hline
\end{tabular}

8. Se ao término dos 10 minutos a qualidade do bloqueio não estivesse satisfatória, seria realizado complementação mais adequada para cada caso.

Foram avaliados:

- Os tempos para instalação do bloqueio motor nos dois grupos.

- Necessidade de complementação anestésica.

- Intensidade da dor durante e ao final do procedimento, pela escala analógica visual de dor (0 a 10) (Quadro 3).

A análise estatística foi realizada pelo método não paramétrico de Mann-Whitney para avaliar a diferença entre os grupos em relação ao tempo de bloqueio, considerando um $\mathrm{p} \leq 0,05$. Para verificar se existe relação entre os grupos e o escore da dor, foi utilizado o teste Qui-quadrado, e nos casos de valores abaixo de 5 realizou o teste exato de Fisher ( $\leq \leq 0,05)$.

\section{RESULTADOS}

Foram estudados 57 pacientes, submetidos à cirurgia de catarata sob anestesia peribulbar, em regime ambulatorial, sendo 31 do sexo masculino $(54,4 \%)$ e 26 do sexo feminino $(45,6 \%)$, e idades variando entre 45 e 89 anos, com média de $67,73( \pm 10,65)$.

$\mathrm{O}$ grupo da ropivacaína associada a hialuronidase (Grupo C) teve 29 pacientes, e o grupo da ropivacaína sem hialuronidase (Grupo S) 28 pacientes.

Os grupos se mostraram homogêneos em relação a sexo, idade e estado físico.

O tempo decorrido entre a realização da anestesia até atingir o escore menor ou igual a três, na escala de Nicoll, foi o utilizado para análise estatística. O tempo médio para se atingir o bloqueio satisfatório no grupo $\mathrm{C}$ foi de 4,07 minutos $( \pm 3,24)$, variando entre 2 e 10 minutos. No grupo $S$, o tempo médio foi de 5,03 ( $\pm 3,28)$, variando entre 2 e 10 minutos. Não foi obser-

\begin{tabular}{|lrrrr|}
\hline \multicolumn{4}{|c|}{ Tabela 1. Tempo de instalação do bloqueio } \\
$\begin{array}{l}\text { Tempo } \\
\text { (em min) }\end{array}$ & $\begin{array}{c}\text { Ropivacaína } \\
\mathbf{n = 2 8}\end{array}$ & $\begin{array}{r}\text { Ropi }+ \text { hialuronidase } \\
\mathbf{n = 2 9}\end{array}$ \\
2 & 12 & $(42,9 \%)$ & 18 & $(62,1 \%)$ \\
4 & 5 & $(60,7 \%)$ & 3 & $(72,4 \%)$ \\
6 & 4 & $(75,0 \%)$ & 1 & $(75,9 \%)$ \\
8 & 2 & $(82,1 \%)$ & 2 & $(82,8 \%)$ \\
10 & 2 & $(89,3 \%)$ & 3 & $(93,1 \%)$ \\
Complementação & $3(100,0 \%)$ & $2(100,0 \%)$ \\
$\begin{array}{l}\text { Os dados entre parênteses expressam a porcentagem acumulada do número } \\
\text { de pacientes }\end{array}$
\end{tabular}




\begin{tabular}{|c|c|c|c|c|c|c|c|c|}
\hline Grupo & 0 & 1 & 2 & 3 & 4 & 5 & 6 & 7 \\
\hline Com & $16(57,1 \%)$ & $1 \quad(3,6 \%)$ & $3(10,7 \%)$ & $5(17,9 \%)$ & $1(3,6 \%)$ & $1(3,6 \%)$ & $0(0 \%)$ & 1 (3,6\%) \\
\hline Sem & $20(69,0 \%)$ & $3(10,7 \%)$ & $2 \quad(6,9 \%)$ & $3(10,7 \%)$ & $0(0,0 \%)$ & 1 (3,6\%) & $0(0 \%)$ & $0(0,0 \%)$ \\
\hline
\end{tabular}

vada diferença estatisticamente significativa entre os dois grupos $(\mathrm{p}=0,146)$ (Tabela 1$)$.

No grupo da ropivacaína com hialuronidase $57,14 \%$ dos pacientes não referiram dor durante o procedimento (nota zero), enquanto no grupo sem hialuronidase $68,97 \%$. Comparando-se os grupos $\mathrm{C} \mathrm{e} \mathrm{S} \mathrm{quanto} \mathrm{ao} \mathrm{escore} \mathrm{de} \mathrm{dor,} \mathrm{não} \mathrm{houve}$ diferença estatisticamente significativa $(\mathrm{p}=0,77)$ (Tabela 2$)$.

Em cinco casos (2 pacientes do grupo $\mathrm{C}$ e 3 do grupo $\mathrm{S}$ ) houve necessidade de complementação anestésica após 10 minutos de avaliação: 2 casos com retrobulbar e 3 com parabulbar.

Foram constatados dois casos de bradicardia intra-operatória, em pacientes não cardiopatas, no grupo sem hialuronidase: uma revertida espontaneamente, e a outra necessitando de atropina $0,5 \mathrm{mg}$, sem maiores conseqüências.

\section{DISCUSSÃO}

Em oftalmologia, a ropivacaína vem sendo utilizada na realização de bloqueio peribulbar, em concentrações de $0,75 \%$ e $1 \%{ }^{(6-9)}$. Estudos comparativos com a solução anestésica tradicionalmente utilizada: bupivacaína associada a lidocaína, mostraram instalação da acinesia estatisticamente mais rápida no primeiro e quinto minutos no grupo da ropivacaína ${ }^{(9-11)}$. Após 15 minutos, o bloqueio sensorial e motor se mostrou semelhante nos dois grupos.

A dose de hialuronidase utilizada varia de $7,5 \mathrm{UTR} / \mathrm{ml}$ a 300 UTR/ml, sendo discutível sua ação adjuvante na anestesia peribulbar $^{(4-5,11-12)}$. Outro estudo ${ }^{(13)}$, a exemplo deste trabalho, não mostrou redução significativa do tempo de instalação do bloqueio quando utilizada hialuronidase na dose de $25 \mathrm{UTR} / \mathrm{ml}$.

Outros autores ${ }^{(14)}$ compararam 3 grupos de pacientes submetidos à anestesia peribulbar com Bupi $0,75 \%+$ Lido $2 \%$, com hialuronidase nas seguintes concentrações: $0 \mathrm{UTR} / \mathrm{ml}$ (A), $50 \mathrm{UTR} / \mathrm{ml}$ (B) e $150 \mathrm{UTR} / \mathrm{ml}$ (C), constatando um tempo de acinesia mais rápido no grupo $\mathrm{C}$ e menor necessidade de complementação, porém, sem diferença estatística. Em estudo recente $^{(4)}$, encontrou-se resultados semelhantes, utilizando $300 \mathrm{UTR} / \mathrm{ml}$ de hialuronidase. Não havendo consenso quanto à concentração eficaz da hialuronidase nas anestesias peribulbares, neste estudo optou-se por $100 \mathrm{UTR} / \mathrm{ml}$.

O grupo que utilizou hialuronidase mostrou uma instalação mais rápida do bloqueio (Nicoll $\leq 3$ ), porém não sendo significativo, provavelmente pelas características farmacológicas da ropivacaína de apresentar um reduzido tempo de instalação do bloqueio ${ }^{(9-10,12)}$. A necessidade de complementação após 10 minutos e o escore de dor foram semelhantes nos dois grupos, não mostrando vantagens na associação da hialuronidase.

Nas condições deste estudo, o uso da hialuronidase associada à ropivacaína em bloqueios peribulbares não mostrou redução do tempo de latência ou melhora da qualidade da anestesia.

\section{ABSTRACT}

Purpose: To evaluate the onset time and quality of peribulbar anesthesia with $1 \%$ ropivacaine associated or not with hyaluronidase $100 \mathrm{TRU} / \mathrm{ml}$ for cataract extraction. Methods: Prospective, randomized, double-blind and controlled study including fifty-seven patients, scheduled to undergo peribulbar anesthesia for cataract extraction, allocated to two groups. Group C: $1 \%$ ropivacaine with addition of $100 \mathrm{TRU} / \mathrm{ml}$ hyaluronidase, and Group S 1\% ropivacaine, without hyaluronidase. The onset time for globe akinesia was studied at intervals of 2 minutes, using Nicoll's score. We evaluated pain by analogic score during the surgery and the necessity of complementing the anaesthesia. The peribulbar block was considered satisfactory when the Nicoll's score was less than 4. Results: The mean time of onset of block in group C was 4.07 minutes $( \pm 3.24)$, and in group $S 5.03( \pm 3.28)$. There was no statistically significant difference between the groups. Both were similar regarding pain score, no pain was observed in $57.14 \%$ of group C, and in $68.97 \%$ of group $\mathrm{S}$. The supplementary anesthetic was necessary in 2 cases of group $\mathrm{C}$ and in 3 cases of group $\mathrm{S}$. Two cases of bradycardia (heart rate $<50 \mathrm{bpm}$ ) were observed during the surgery, and in one case administration of atropine IV was necessary. Conclusion: $1 \%$ ropivacaine provided a good quality of anesthesia for cataract extraction, with a faster onset of action in the group with hyaluronidase $100 \mathrm{iu} / \mathrm{ml}$, although without significant difference.

Keywords: Cataract extraction; Ropivacaine/administration \& dosage; Hyaluronoglucosaminidase/administration \& dosage; Local anesthesia/methods; Comparative study; Efficacy

\section{REFERÊNCIAS}

1. McClure JH. Ropivacaine. Br J Anaesth 1996;76:300-7.

2. Knudsen K, Beckman Suurkula M, Blomberg S, Sjövall J, Edvardson N. Central nervous and cardiovascular effects of i.v. infusions of ropivacaine, bupivacaine and placebo in volunteers. Br J Anaesth 1997;78:507-14.

3. Wong DH. Regional Anaesthesia for intraocular surgery. Can J Anesth 1993;40:635-57.

4. Dempsey GA, Barret PJ, Kirby IJ. Hyaluronidase and peribulbar block. Br J Anaesth 1997;78:671-4. 
5. Bowman RJ, Newman DK, Richardson EC, Callear AB, Flanagan DW. Is hyaluronidase helpful for peribulbar anaesthesia? Eye 1997;11:385-8.

6. Corke PJ, Barker J, Cammack R. Comparison of $1 \%$ Ropivacaine and a mixture of $2 \%$ lignocaine and $0.5 \%$ bupivacaine for peribulbar anaesthesia in cataract surgery. Anaesth Intensive Care 1999;27:249-52.

7. Gioia L, Prande E, Codenott $\mathrm{M}$ et al. Peribulbar anaesthesia with either $0.75 \%$ Ropivacaine or a $2 \%$ Lidocaine and $0.5 \%$ Bupivacaine mixture for vitreoretinal surgery: A double-blinded study. Anesth Analg 1999;89:739-42.

8. Nicholson G, Sutton B, Hall GM. Ropivacaine for peribulbar anesthesia. Reg Anesth Pain Med 1999;24:337-40.

9. McLure HÁ, Rubin AP, Westcott M et al. A comparison of $1 \%$ ropivacaine with a mixture of $0.75 \%$ bupivacaine and $2 \%$ lignocaine for peribulbar anaesthesia. Anaesthesia 1999;54:1178-82.
10. Nociti JR, Serzedo PS, Zuccolotto EB, Cagnolati CA, Nunes AM. Ropivacaine in Peribulbar block: a comparative study with bupivacaine. Acta Anaesthesiol Scand 1999;43:799-802.

11. Gillart T, Barrau P, Bazin JE, Roche G, Chiambaretta F, Schoefler P. Lidocaine plus ropivacaine versus lidocaine plus bupivacaine for peribulbar anaesthesia by single medial injection. Anesth Analg 1991;89:1192-6.

12. Simonetti MPB. Ropivacaína: Estado atual e perspectivas futuras. Rev Bras Anestesiol 1995;45:131-40.

13. Prosser DP, Rodney GE, Mian T, Jones HM, Khan MY. Re-evaluation of hyaluronidase in peribulbar anesthesia. Br J Ophtalmol 1996;80:827-30.

14. Brydon CW, Basler M, Kerr WJ. An evaluation of two concentrations of hyaluronidase for supplementation of peribulbar anaesthesia. Anaesthesia 1995;50:998-1000.

\section{Simpósio Infernacional de Órbita}

\section{Reunião Anual do Centro de Estudos Oftalmológicos "Cyro de Rezende"}

\section{Avanços em cirurgias do segmente anterior}

06 a 08 de Dezembro de 2002
RIBEIRÃO PRETO - SP
Hotel Stream Palace

INFORMAÇÕES: Tels.: (16) 602-2523/2862/2863 com as Sras Amélia, Rita ou Cecília 